\title{
Motion Detection by Quantum-Dots-Based Velocity-Tuned Filter
}

\author{
Woo Hyung Lee and Pinaki Mazumder, Fellow, IEEE
}

\begin{abstract}
Motion estimation is a major computational task in real-time vision circuits and artificial retinas that require energyefficient, high-speed, and microminiaturized circuitry. Traditionally, the motion estimation is made by means of velocity-tuned filters (VTFs), a class of spatiotemporal signal processing circuitry. However, conventional VTFs have limitations in area, power, and speed for real-time motion computation because they employ bulky and slow analog circuitry. In this paper, we propose a nanoscale VTF that employs quantum dot arrays to perform temporal filtering to track moving and stationary objects. The new velocity-tuned filter is not only amenable for nanocomputing, but also superior to other VTFs in terms of area, power, and speed. We also show that the proposed nanoarchitecture for VTF is asymptotically stable in the specific region where $f^{\prime}\left(S_{n, m}\right) \geq 0$.
\end{abstract}

Index Terms-Nanoelectronic, quantum dot, resonant tunneling diode (RTD), velocity-tuned filter (VTF).

\section{INTRODUCTION}

I $\mathrm{T}$ IS well known that real-time vision machine application tasks are computationally intensive and require complex, costly resources. In addition, certain specific tasks such as biorobots and biomedical applications put additional constraints on the overall system in terms of its size, power consumption, shock resistance, and manufacturing cost. A real-time vision machine requires motion computation involving a large number of computations and many computational resources. An attractive solution to the problems is to use parallel image-processing architectures [1], [2].

Since velocity-tuned filter (VTFs) are one of the main parts of motion computation, they require a compact area and low power consumption. The VTF can be combined with pattern recognizers, optical flow sensors, and noise removers to create realtime vision machines. Even though VTFs using spatiotemporal derivatives and Reichardt correlation detectors have been studied, they cannot provide sufficient area compactness, low power consumption, and/or speed [3], [4]. These problems can be attributed to limitations of conventional analog circuits that cannot achieve high performance for the real-time vision machine.

Among nanoelectronic devices, a quantum dot structure on a surface of a doubled barrier resonant tunneling diode (RTD) shows an excellent performance in terms of area, power consumption, and speed. Since being proposed by several re-

Manuscript received September 22, 2006; revised May 14, 2007 and September 9,2007 . This work was supported in part by the Office of Naval Research. The review of this paper was arranged by Associate Editor K. Likharev.

The authors are with the Department of Electrical Engineering and Computer Science, University of Michigan, Ann Arbor, MI 48109 USA (e-mail: leewh@eecs.umich.edu; mazum@eecs.umich.edu).

Color versions of one or more of the figures in this paper are available online at http://ieeexplore.ieee.org.

Digital Object Identifier 10.1109/TNANO.2007.915019

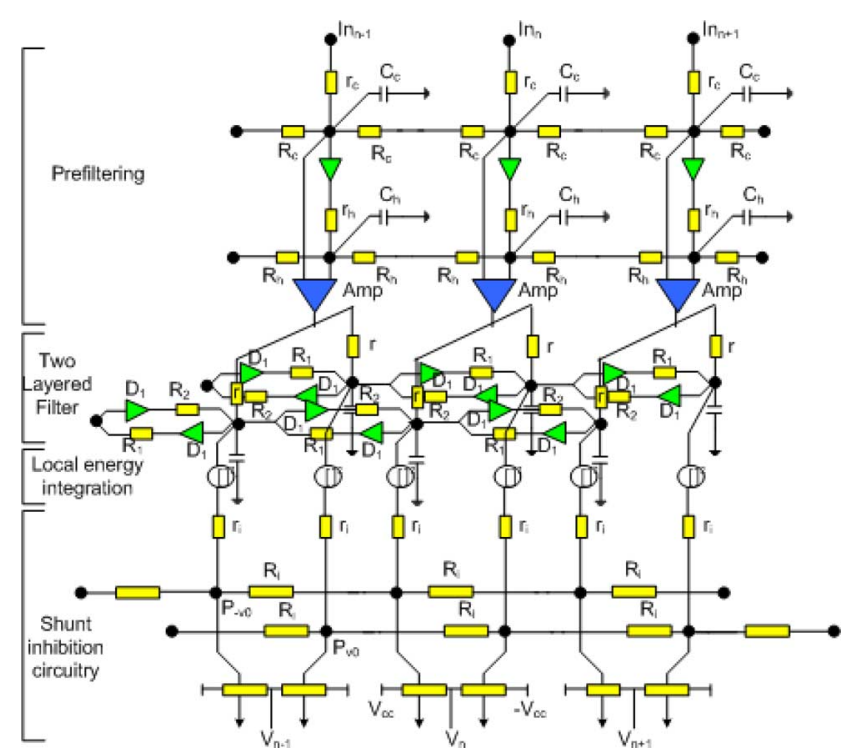

Fig. 1. Conventional VTF that consists of prefiltering, two layered filters, local energy integration, and shunting inhibition circuitry.

searchers [1], [2], their applications have been confined to Boolean logic or image processing.

We propose a new type of VTF using nanoelectronic devices. The proposed VTF achieves performance in area, power, and speed superior to other conventional VTFs by taking advantage of the efficient parallel processing capability of the quantum dots structure.

The remainder of this paper is organized as follows. Section II discusses the proposed quantum-dots-based VTFs. In Section II, the electrical characteristics of RTD and conventional VTFs are addressed to help to comprehend the proposed VTFs. Section III studies the system analysis of the proposed VTFs in speed, power, and stability. Finally, Section IV presents our conclusions.

\section{QUANTUM-DOTS-BASED VTFS}

\section{A. Conventional VTF}

Fig. 1 shows a conventional VTF. The conventional VTF consists of preamplifiers, filters, multipliers, and differential amplifiers. A preamplifier consists of a prefiltering part and an amplification part. In the prefiltering part, the input signal $I n_{n}$ comes from photo detectors that are not shown in Fig. 1. After the input signal is amplified by the preamplifiers, the input signal passes through the two layered filters: the receptor layer and the horizontal cell layer, respectively. The main function of the receptor layer is to improve the SNR. The second layer calculates a spatiotemporal average of the receptor output. The 
combined function of the two layered filter is a spatiotemporal bandpass filter.

After prefiltering the signal from the optical flow, the filtered signal is amplified for providing inputs to two layered filters. The signal is then filtered with the two layered filters that allow the signals to have different values based on the velocities. The filtered signal is then magnified with local energy integration, and the output is linearized with the difference between signals using shunting inhibition circuitry.

The local energy integration can be written in a mathematical form as

$$
\begin{aligned}
P(\Delta v) & =\int \Gamma_{s}\left(f_{x}\right) d f_{x} \\
& =\int \Gamma_{e}\left(f_{x}\right)\left|G\left(f_{x}\right)\right|^{2} d f_{x} \\
& =\Gamma \int\left|G\left(f_{x}\right)\right|^{2} d f_{x}
\end{aligned}
$$

where $V_{x}$ and $G\left(f_{s}\right)$ are the input velocity and the frequency response, and $\Gamma$ and $\Delta v_{0}$ are constants [5]. The VTF needs the shunting inhibition circuitry to linearize the output. The function of the shunting inhibition is mathematically written as

$$
V_{\mathrm{out}}=\frac{G_{+}-G_{-}}{G_{+}+G_{-}} V_{c c}=\frac{P_{V_{0}}-P_{-V_{0}}}{P_{V_{0}}+P_{-V_{0}}} V_{c c} .
$$

Since the conventional VTFs use analog circuitry to compute (1) and (2), the conventional VTFs are not well suited for realtime motion computation circuits for biorobots or biomedical applications, which require high performance in area, power, and speed. Hence, the conventional VTFs hardly meet the condition for real-time motion estimation for those applications.

To implement the real-time motion estimation, we employ zero dimensional RTDs that are implemented by quantum dots on the RTDs. Since the incident light is prefiltered, and then amplified as an electric signal in the first part in Fig. 1, we focus on the remaining part to improve the performance of the VTF. The quantum dots on the RTDs are used to replace the filtering part, the local energy integration part, and the shunt inhibition part. These functions can be implemented by using the negative resistance characteristic and area compactness of the quantum dots on the RTDs.

\section{B. Resonant Tunneling Diode}

Since the RTD was introduced by Esaki et al [6], [7], it has been applied to various types of circuitry [8]. The main characteristic of the RTD is the negative differential resistance (NDR). This characteristic originates from its heterostructure with a low-bandgap quantum well between high-bandgap materials. The thickness and the width of the RTD are required to be fabricated in the order of several nanometers with epitaxial deposition techniques. The low-bandgap quantum well is quantized, resulting in discretized energy levels in the quantum well. Fig. 2(a) shows the modeling of the RTD $I-V$ characteristic from experimental results. The fabricated RTD describes a peak-to-valley current ratio (PVCR) of 13 with a peak voltage

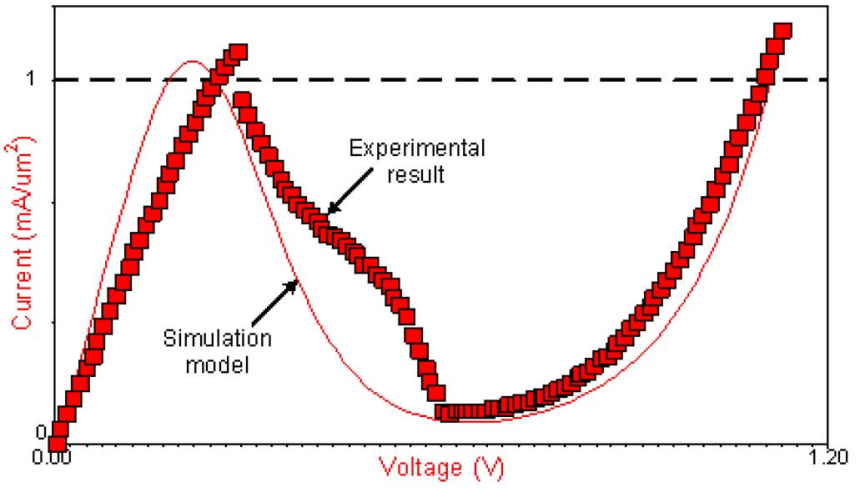

(a)

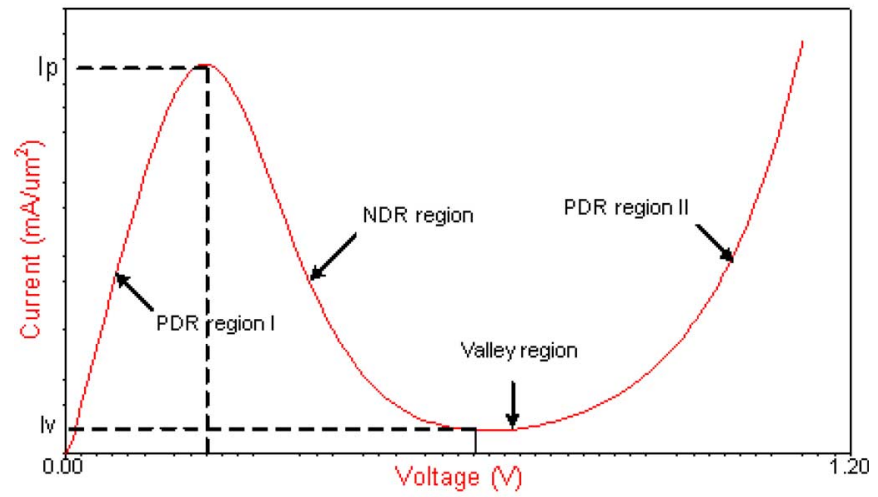

(b)

Fig. 2. Modeling of RTD based on experimental result. (a) Experimental result and simulation model, (b) Simulation model of RTD with several regions.

(VP) of $0.28 V$ at room temperature. To model this $I-V$ curve, factors that affect the conduction of RTD need to be examined.

The conduction of the RTD consists of two parts. One is conduction due to resonant tunneling and the other is conduction from diode conduction. The NDR results from the resonant tunneling conduction effect [9]-[11]. Using a physics-based model suggested by Schulman et al., the resonant tunneling current of RTD is modeled as a summation of the resonant tunneling effect $J_{1}$ and diode conduction effect $J_{2}$, given by

$$
\begin{aligned}
J(V)= & J_{1}(V)+J_{2}(V) \\
J_{1}(V)= & \frac{q m * k T \Gamma}{4 \pi^{2} \hbar^{3}} \ln \left(\frac{1+e^{\left(E_{F}-E_{r}+n_{1} q V / 2\right) / k T}}{1+e^{\left(E_{F}-E_{r}-n_{1} q V / 2\right) / k T}}\right) \\
& \times\left(\frac{\pi}{2}+\arctan \left(\frac{E_{r}-n_{1} q V / 2}{\Gamma / 2}\right)\right) \\
J_{2}(V)= & H\left(e^{n_{2} q V / k T-1}\right)
\end{aligned}
$$

where $E_{F}$ is the Fermi energy, $E_{r}$ is the energy of the resonant level, $\Gamma$ is the resonant width, and $n_{1}$ and $n_{2}$ are model parameters. These parameters are often obtained empirically, and affect the slope of the curve in Fig. 2(b).

The resonant tunneling occurs when the applied voltage across the diode is aligned to one of the quantized energy levels in the quantum well, as shown in positive differential-resistance (PDR) region $I$ in Fig. 2(b). However, when the applied voltage is increased to be misaligned to the quantized energy level, 
the conduction is decreased, as shown in the NDR region in Fig. 2(b). The current subsequently increases as conduction through higher energy states becomes possible, as shown in the PDR region in Fig. 2(b). This characteristic enables the circuit switch fast and self-latching or bistable. Using this characteristic, a wide class of circuit applications, for instance, highspeed circuits, low-power-delay product circuits, and multivalued logic, can be implemented.

\section{Proposed VTF}

The proposed VTF consists of quantum dots on a RTD substrate. The quantum dots are connected with resistive wires and diodes. Each cell is connected to four neighboring cells and one output quantum dot. The output quantum dot needs a static current source that is vertically connected to the RTD substrate. Conventional VTFs require local energy integration and shunting inhibition circuits to magnify the output difference. Our filter structure does not employ these circuits, and instead, uses the RTD to magnify the filtered output differences. Through the differential resistance characteristic of the RTD, the final value is determined by the RTD's $I-V$ characteristic and the external current source. In the proposed VTF, the output of the filter makes the RTD device operate when the output of the filter is higher than $1.4 \mathrm{~V}$. This threshold voltage can be obtained as

$$
V_{\mathrm{th}}=V_{d}+\frac{V_{p}+V_{v}}{2}
$$

where $V_{d}$ is the diode junction voltage, and $V_{p}$ and $V_{v}$ are the peak voltage and the valley voltage, respectively, in the RTD $I-V$ curve.

Fig. 3 shows the schematic diagrams of the proposed VTF's side view and top view. The analysis of the proposed VTF requires a new state equation. This new state equation of the proposed structure is obtained by modifying the state equation of the diffusion circuit in (7), where $X_{n, m}, S_{n, m}$, and $v$ represent the input voltage, the output voltage, and the velocity, respectively. Also, $\gamma$ and $\tau$ are defined in (8) and (9). Here, $\gamma$ is related with conductance between quantum dots in the filtering part and $\tau$ affects the processing time of the filter. Also, $v_{x 0}$ and $v_{y 0}$ represent the tuning velocity in the $x$-direction and the $y$-direction, respectively

$$
\begin{aligned}
& \tau \frac{d S_{n, m}(t)}{d t}= X_{n, m}(t)-S_{n, m}(t) \\
&+\left(\gamma^{2}+\frac{v_{x 0} \tau}{2}\right)\left(S_{n-1, m}(t)-S_{n, m}(t)\right) \\
&+\left(\gamma^{2}+\frac{v_{y 0} \tau}{2}\right)\left(S_{n, m-1}(t)-S_{n, m}(t)\right) \\
&+\left(\gamma^{2}-\frac{v_{x 0} \tau}{2}\right)\left(S_{n+1, m}(t)-S_{n, m}(t)\right) \\
&+\left(\gamma^{2}-\frac{v_{y 0} \tau}{2}\right)\left(S_{n, m+1}(t)-S_{n, m}(t)\right) \\
& \gamma^{2}= \frac{r T / q I_{s}\left(e^{q V_{d} / k T}-1\right)+R}{\tau=} \\
& \tau C .
\end{aligned}
$$

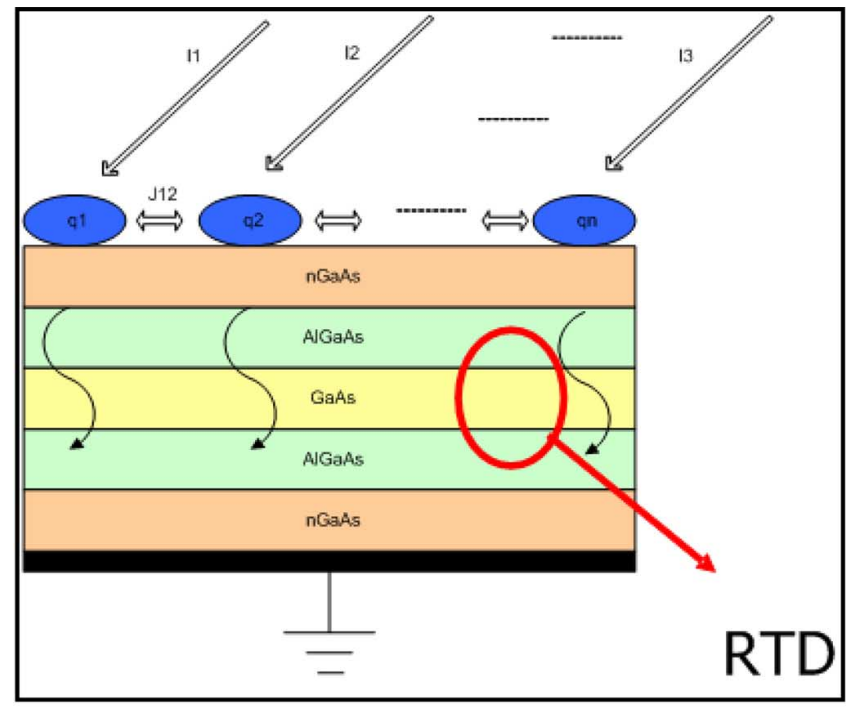

(a)

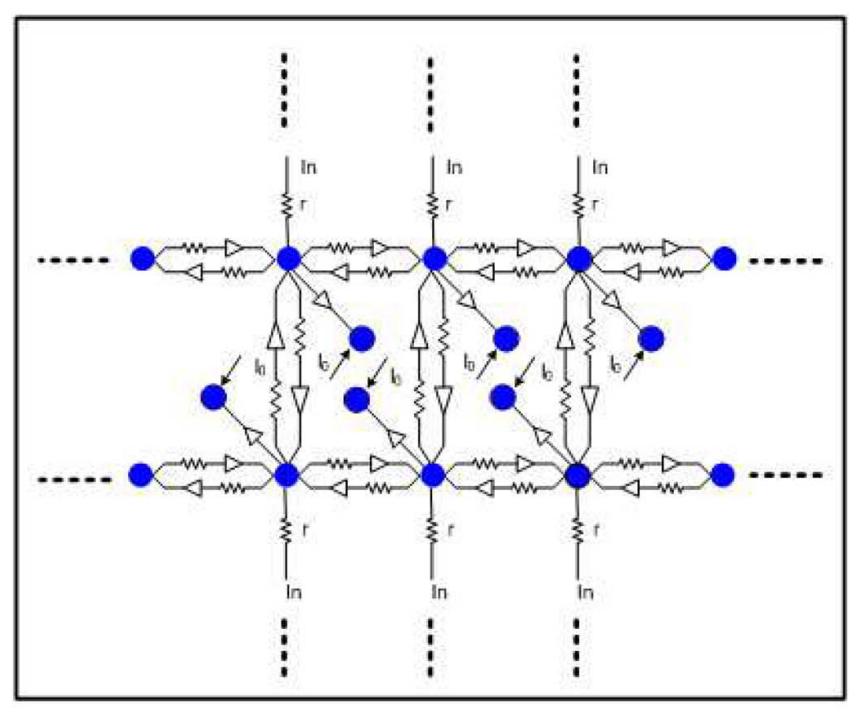

(b)

Fig. 3. Proposed VTF with quantum dots structure on the RTD substrate. (a) Side view. (b) Top view.

Considering the RTD, the state equation can be obtained as

$$
\begin{aligned}
\frac{d}{d t} q_{n, m}= & C_{n, m} \frac{d S_{n, m}}{d t} \\
= & -f\left(S_{n, m}(t)\right)+h\left(S_{n, m}\right) \\
f\left(S_{n, m}(t)\right)= & F\left(\exp \left(n_{2} S_{n, m} q / k T\right)-1\right) \\
& +A \ln \left[\frac{1+e^{\left(\left(B-C+n_{1} S_{n, m}\right) q / k T\right)}}{1+e^{\left(\left(B-C+n_{1} S_{n, m}\right) q / k T\right)}}\right] \\
& \times\left[\frac{\pi}{2}+\arctan \left(\frac{C-n_{1} S_{n, m}}{D}\right)\right] \\
& +H\left(e^{n_{2} e V / k T-1}\right) \\
h\left(S_{n, m}(t)\right)= & C e l l(k, l) \in N_{r}(n, m) \\
& \times\left(e^{q\left(N_{r}(n, m)-I_{r} R-S_{n, m}\right) / k T}-1\right) I_{s}
\end{aligned}
$$




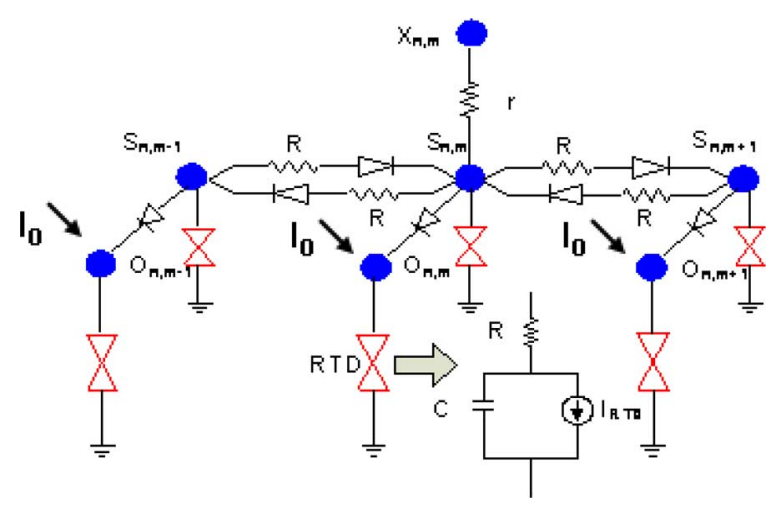

Fig. 4. Circuit model of a basic cell of the proposed VTF.

where

$$
\begin{aligned}
& G\left(N_{r}(n, m), \overrightarrow{v(t, x, y)}\right) \\
& =\left\{\begin{array}{ll}
\gamma^{2}+\frac{\vec{v}(t, x, y) \tau}{2} & \cos \theta\left(\overrightarrow{v(t, x, y)}, \overrightarrow{N_{r}(n, m) S_{n, m}}\right)=0 \\
\gamma^{2}-\frac{\vec{v}(t, x, y) \tau}{2} & \cos \theta\left(\overrightarrow{v(t, x, y)}, \overrightarrow{N_{r}(n, m) S_{n, m}}\right) \neq 0
\end{array}\right\}
\end{aligned}
$$

and $N_{r}(n, m)$ is an $r$-neighborhood of a cell $\operatorname{Cell}(n, m)$ and is defined by

$$
N_{r}(n, m)=\left\{\begin{array}{l}
\operatorname{Cell}(k, l) \mid \max \{|k-n|,|l-m|\} \leq r, \\
1 \leq k \leq N ; \quad 1 \leq l \leq M
\end{array}\right\}
$$

where $I_{n, m}$ is the bias current and $f\left(S_{n, m}\right)$ describes the $I-$ $V$ characteristic of the RTD with physical model parameters ,, $A, B, C, D, F, n_{1}$, and $n_{2} ; h\left(S_{n, m}\right)$ represents the current from neighboring cells to $S_{n, m}$ where $I_{s}$ is the saturation current in the diode.

The relationship between $S_{n, m}$ and $O_{n, m}$ can be described by combining (10) and the following:

$$
g\left(O_{n, m}(t)\right)=\left(e^{q\left(S_{n, m}-O_{n, m}\right) / k T}-1\right) I_{s} .
$$

Using these equations, we obtain a new state equation as

$$
C_{n, m} \frac{d O_{n, m}}{d t}=-f\left(O_{n, m}(t)\right)+g\left(O_{n, m}(t)\right)+I_{n, m}(t)
$$

which represents the VTF. The schematic diagram of the VTF for a basic cell is described in Fig. 4. Figs. 5 and 6 describe experimental results of the proposed VTF. In Figs. 5 and 6, the dark-round-shaped dots represent input impulse signals. Observing signals based on the time sequence, the moving object, and the standing object are divided by time T. Hence, $T=2$ represents the next time to $T=1$ in the sampling clock sequence. In Fig. 5, the standing object $\left(S_{4}\right)$ is amplified in the output $O_{4}$, while other moving objects from $S_{2}$ to $S_{1}$ hold low output values in $O_{1}$. In Fig. 6, the moving object from $S_{2}$ to $S_{1}$ in the left direction is latched to the high value in $O_{1}$, while the standing object $S_{4}$ is latched to the low output value in $O_{4}$. In Fig. 7, the left figure is the moving input. The first row represents an object moving with a velocity of 2 pixels/s to the right. The second row shows an object with a velocity of $1 \mathrm{pixel} / \mathrm{s}$ to the right. The third row represents a nonmoving object. The fourth row shows an object with a velocity of $1 \mathrm{pixel} / \mathrm{s}$ to the

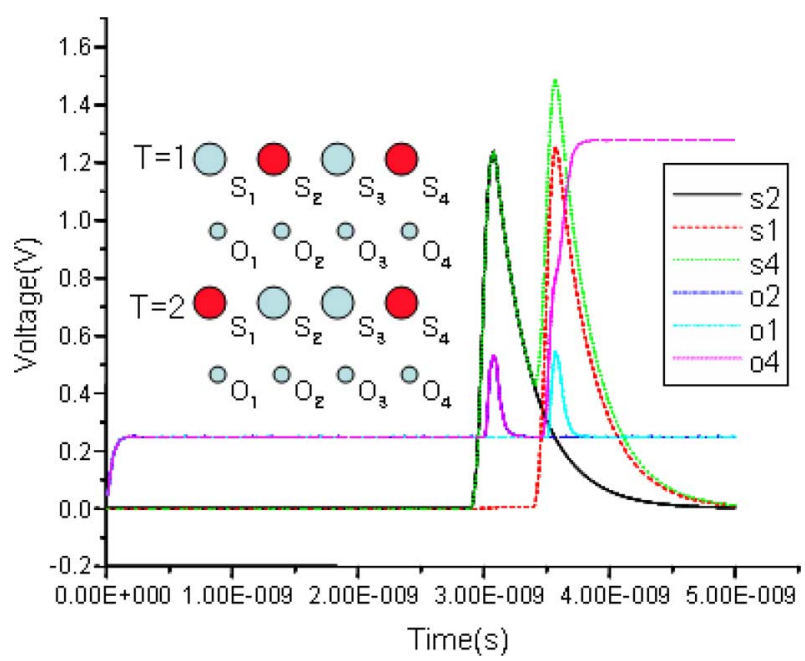

Fig. 5. HSPICE simulation result for VTF with velocity 0 .

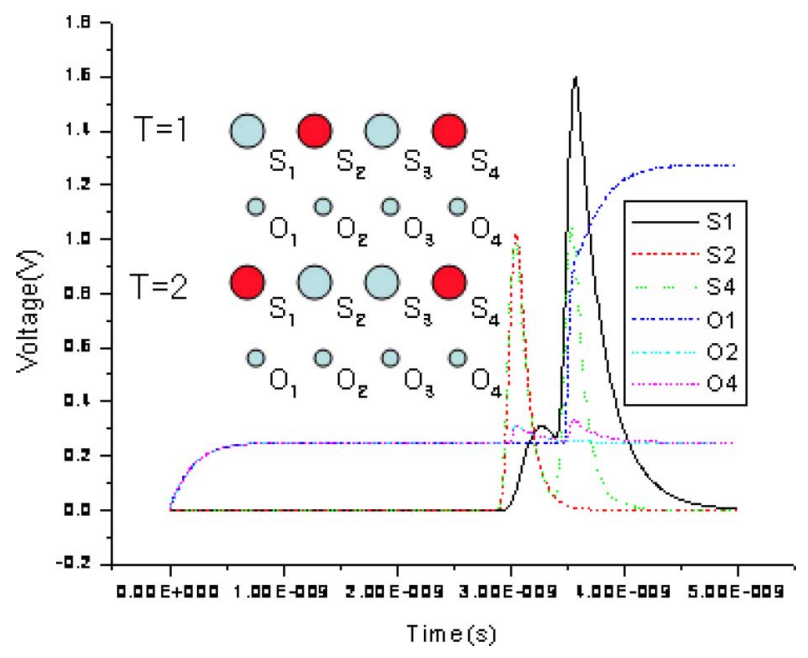

Fig. 6. HSPICE simulation result for VTF with velocity 1.

left. Finally, the fifth row represents an object with a velocity of 2 pixels/s to the left. The right sides of Figs. 7 and Fig. 8 show the output of the filter. In Fig. 7, only the target object, which does not move has a high output value shown with red and yellow colors. Therefore, the nonmoving object can be detected, as shown in Fig. 7(b). In Fig. 8, the moving object with a velocity of $1 \mathrm{pixel} / \mathrm{s}$ to the left has a high output value. Hence, the object with a velocity of $1 \mathrm{pixel} / \mathrm{s}$ to the left can be detected, as shown in Fig. 8(b). The velocity to be filtered can be chosen by controlling the resistance values between the quantum dots. Based on (7), we control the resistance value to filter objects with a certain velocity. The output results in Fig. 8(b) can be explained using (15).

\section{System ANALYSis}

\section{A. Delay Analysis of VTF}

In this section, we investigate the delay of the input signal to the output node. To analyze the signal delay, we regard our VTF as an $R C$ network in the form of a tree. To examine the system as an $R C$ tree, we simplify our VTF as shown in Fig. 9. In Fig. 9, 


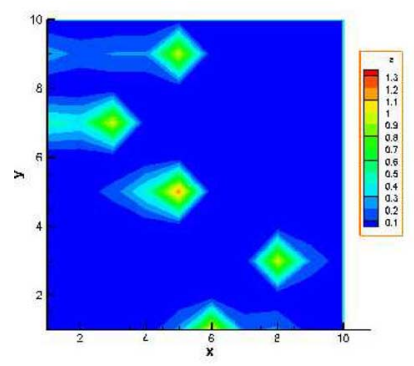

(a)

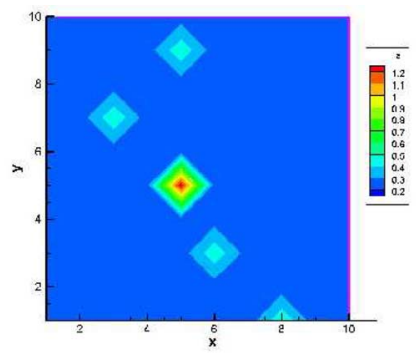

(b)
Fig. 7. Experimental result of VTF for a velocity of 0 pixel/second. (a) Input moving objects. (b) Output filtered objects.

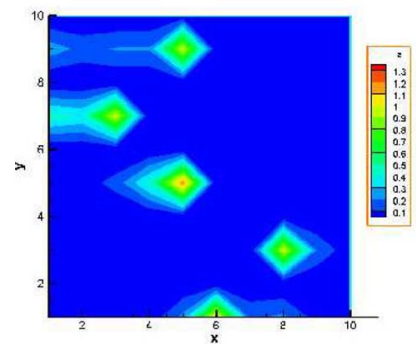

(a)

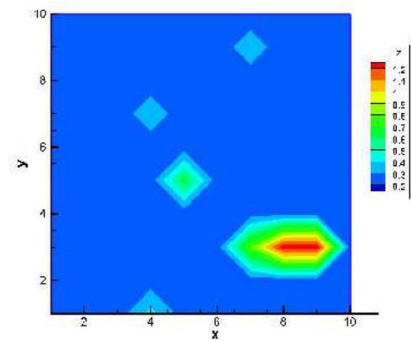

(b)
Fig. 8. Experimental result of VTF for a velocity of 1 pixel/second. (a) Input moving objects. (b) Output filtered objects.

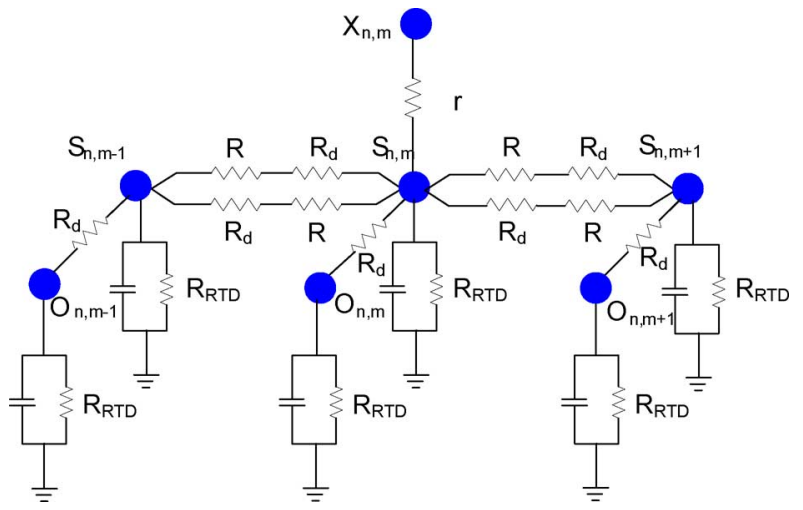

Fig. 9. $\mathrm{RC}$ tree representation of the proposed VTF.

we represent the VTF only with capacitors and resistors. Hence, the following equation always holds for the output node $O_{n, m}$ :

$$
\int_{0}^{\infty} V_{O_{n, m}}(t) d t=\sum_{k=1}^{N} C_{k} S_{k}(0) R_{O_{n, m}, k}
$$

where

$$
\begin{aligned}
R_{O_{n, m}, k}= & \sum R_{j} \Rightarrow\left(R _ { j } \in \left[\operatorname{path}\left(O_{n, m} \rightarrow X_{n, m}\right)\right.\right. \\
& \left.\left.\bigcap \operatorname{path}\left(k \rightarrow X_{n, m}\right)\right]\right) .
\end{aligned}
$$

If we assume that the array of filter nodes is a $2 n \times 2 m$ matrix, same as that of the output nodes, the delay of the signal from the input node to the output node in the position of $n, m$ can be

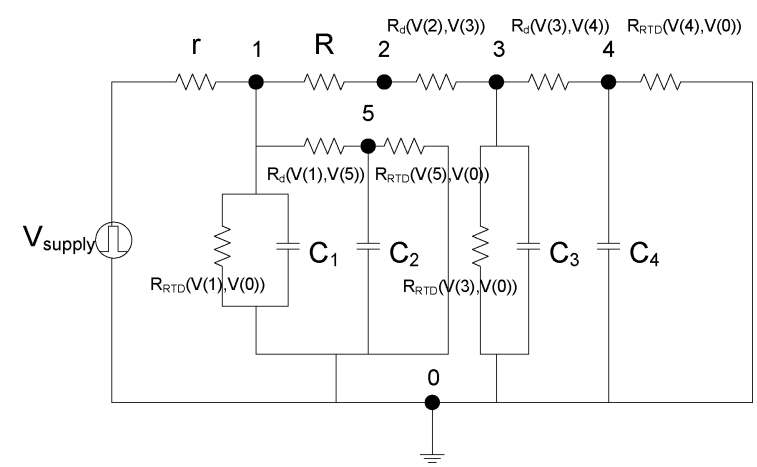

Fig. 10. Equivalent circuit model of an unit cell of VTFs.

represented by

$$
\begin{aligned}
& r \sum_{l=1}^{2 n} \sum_{k=1}^{2 m}\left(C_{S_{k, l}}+C_{O_{k, l}}\right)-r C_{O_{n, m}} \\
& +\left(r+R_{d}(V)+5 R_{\mathrm{rtd}}(V)\right) C_{O_{n, m}}
\end{aligned}
$$

where $R_{\text {rtd }}(V)$ and $R_{d}(V)$ are the resistances with functions of applied voltages across each device. Since we assume that the output is stabilized after $5 \tau$ (time constant) due to the RTD, we add $5 R_{\text {rtd }}(V)$ for the RTD stabilization time on the output. Based on the simulation results in Figs. 5 and 6, the RTD stabilization takes time from 200 to 500 ps in our simulation. Assuming that the capacitances are same for all nodes, (18) can be approximated as

$$
C\left(4 r n \times 4 r m+R_{d}(V)+5 R_{\mathrm{rtd}}(V)\right) .
$$

Given the size of the array, the delay is governed by the input resistance, the diode resistance, and the RTD resistance from (19).

However, since the conventional VTF uses local energy integration and shunt inhibition circuitry after filtering, our VTF is much faster than the conventional VTF. To examine this in detail, the propagation delay of the local energy integration and the shunt inhibition circuitry needs to be investigated. In the circuit implementation, the integration logic consists of multipliers and adders. Using the cutting-edge technology, $0.11 \mu \mathrm{m}$ CMOS standard cell library, the whole processing of those combinational logics takes 4.5-5 ns for $4 \mathrm{~b}$ (one digit) multipliers [12]. Including adders, the processing time will be increased until 7-8 ns, which is around 100 times slower than that of our nanoelectronic circuitry, as shown in Figs. 5 and 6.

\section{B. Power Consumption Analysis of VTF}

Since our proposed VTF has fewer device components than conventional VTFs, the proposed VTF is assumed to consume less power. To analyze the power consumption, we need to partition the VTF into two components. The first part corresponds to the filter part, which includes filtering connections, input connections, quantum dots, and the RTD in Fig. 4, and the second part consists of output interconnections, output quantum dots, and the output RTD in Fig. 4. To calculate the power dissipation, we redraw Fig. 4 as Fig. 10 that excludes the right-hand side of the filters for circuit analysis, assuming that the current flow in the filter area is unidirectional. If we assume that the input 
TABLE I

PERFORMANCE COMPARISON OF VTFs WITH $20 \times 20$ PIXEL IMAGES

\begin{tabular}{cccc}
\hline \hline & Sequential VTF & Conventional network VTF & Proposed VTF \\
\hline Area & $\sim 1000 \mu^{2}$ & $\sim 100 \mu^{2}$ & $\sim 10 \mathrm{~nm}^{2}$ \\
Processing Time & $0.1 \sim 10 \mathrm{~ms}(1 \mathrm{GHz}$ CPU) & $0.1 \sim 10 \mu \mathrm{s}$ & $0.3 \sim 2 n s$ \\
Power & $0.1 \sim 1 \mathrm{~mW}$ & $0.1 \sim 1 \mathrm{~mW}$ & $1 \sim 10 \mu \mathrm{W}$ \\
\hline
\end{tabular}

voltage to node $X_{n, m}$ in Fig. 9 is $V_{\text {supply }}$, which is a pulse signal with a rising and a falling time, the average power consumption of resistor $r$ is given as

$$
\begin{aligned}
P_{r} & =r I^{2}=r\left(Y V_{\text {supply }}\right)^{2}=r\left|\frac{1}{Z}\right|^{2}\left|V_{\text {supply }}\right|^{2} \\
Y & =\frac{1}{Z}
\end{aligned}
$$

where, $Z$ is given by (22), shown at the bottom of the page. In (22),

$$
A=\frac{(1 / j \omega C) R_{\mathrm{RTD}}(V)}{1 / j \omega C+R_{\mathrm{RTD}}(V)}
$$

where $R_{\mathrm{RTD}}(V)$ and $R_{d}(V)$ are functions of voltage $V$ applied across the devices. $R_{\mathrm{RTD}}(V)$ and $R_{d}(V)$ can be obtained from $I-V$ curves when voltages are applied across the devices. The $I-V$ curve of the diode is described by

$$
I=I_{s}\left(e^{q V / k T}-1\right) .
$$

Also, the $I-V$ curve of RTDs is described by (3)-(5). Since $V_{\text {supply }}$ is not a dc source, the voltages across the devices change with time. This calculation can be processed by partitioning the simulation time and applying the equations with each interval repeatedly. The average power consumption of resistor $R$ is given as

$$
\begin{aligned}
P_{R}= & R I_{1}^{2} \\
= & R\left|\frac{A\left(R_{d}(V)+A\right) / 2 A+R_{d}(V)}{\left(R+R_{d}(V)\right)+2 A\left(R_{d}(V)+A\right) /\left(2 A+R_{d}(V)\right)}\right|^{2} \\
& \times\left|\frac{1}{Z}\right|^{2}|V|^{2} .
\end{aligned}
$$

In the case of a diode in the filter parts, the average power consumption is written as

$$
\begin{aligned}
P_{R_{d}(V)}= & R_{d}(V)\left|\frac{A\left(R_{d}(V)+A\right) / 2 A+R_{d}(V)}{R+R_{d}(V)+2 A\left(R_{d}(V)+A\right) / 2 A+R_{d}(V)}\right|^{2} \\
& \times\left|\frac{1}{Z}\right|^{2}|V|^{2} .
\end{aligned}
$$

The output that includes the diode, quantum dot, and RTD consumes power described by

$$
\begin{aligned}
P_{\text {output }}= & \frac{\left|A\left(R_{d}(V)+A\right) / 2 A+R_{d}(V)\right|^{3}}{\left|R+R_{d}(V)+2 A\left(R_{d}(V)+A\right) / 2 A+R_{d}(V)\right|^{2}} \\
& \times\left|\frac{1}{Z}\right|^{2}|V|^{2} .
\end{aligned}
$$

The total power dissipation can be obtained by measuring the current from the power source and multiplying with the supplied voltage. We could obtain the total power dissipation by using the HSPICE simulation that includes the aforementioned calculations. However, since the output part include the external current into the output quantum dot, the power consumption due to the external current needs to be added to the output power consumption. The modified power consumption including the contribution from the external current is given as

$$
P_{\text {output-mod }}=P_{\text {output }}+I_{\text {ext }}^{2} R_{\mathrm{RTD}}(V) .
$$

In detail, the external current can be minimized to minimize the static power consumption by satisfying the condition

$$
I_{v}+I_{\text {leak }}<I_{\text {ext }}<I_{\text {peak }}+I_{\text {leak }}
$$

where the $I_{\text {leak }}, I_{\text {peak }}$, and $I_{v}$ are the leakage current from the capacitance components of the RTDs, the peak current, and the valley current, respectively, as shown in Fig. 2(b). If we apply the current density of $0.5 \mathrm{~mA} / \mu \mathrm{m}^{2}$, we have two stable voltage points 0.15 and $1.1 \mathrm{~V}$. Therefore, the corresponding power dissipations from the external current source are 15 and $110 \mathrm{nW}$. If we apply the experimental values of the RTDs' capacitance of $10^{-15} \mathrm{~F}$ and $V_{\text {supply }}$ of $1.5 \mathrm{~V}$ to the HSPICE simulation, we get the total average power dissipation in the range from 29 to $114 \mathrm{nW}$, depending on output voltages. Hence, if we have high output voltages, the external current is dominant in the total power dissipation; otherwise, it is comparable with dynamic power dissipation. However, the dynamic power dissipation is dominated by the power from the input resistance $r$ and the resistances $R$ in the filter, each of which has much higher value than the other resistive components.

Based on the earlier calculations, Table I gives the performance comparison with the VTFs in [5] and [13]. In terms of area, our VTF is 10-100 times smaller than the other VTFs. As shown in Table I, the processing time of our VTF is three to six orders of magnitude faster than the other VTFs and consumes around 100 times less power than the other VTFs.

\section{Stability of VTF}

Stability is an important issue in designing a system. Since the VTF consists of nonlinear elements, namely quantum dots on an RTD, we need to use appropriate methods for examining the stability of the nonlinear system. One such method is the Lyapunov theorem. To apply the Lyapunov method, we define

$$
Z=r+\frac{\left(R+R_{d}(V)+A\left(R_{d}(V)+A\right) / 2 A+R_{d}(V)\right) A\left(R_{d}(V)+A\right) / 2 A+R_{d}(V)}{R+R_{d}(V)+2 A\left(R_{d}(V)+A\right) / 2 A+R_{d}(V)}
$$


the Lyapunov function $E(t)$ of the VTF as

$$
\begin{aligned}
E(t)= & \frac{1}{2} \sum_{n, m} h\left(S_{n, m}(t)\right) h\left(S_{k, l}(t)\right) \\
& +\frac{1}{2} \sum_{n, m} f^{2}\left(S_{n, m}(t)\right) \\
& -\sum_{n, m} I_{n, m} S_{n, m}(t) .
\end{aligned}
$$

Considering that $f\left(S_{n, m}\right)$ is a nonlinear equation, $E(t)$ can be described by

$$
\begin{aligned}
E(t)= & \frac{1}{2} \sum_{n, m} h\left(S_{n, m}(t)\right) h\left(S_{k, l}(t)\right) \\
& +\sum_{n, m} \int_{0}^{S_{n, m}} f\left(S_{n, m}\right) d S_{n, m} \\
& -\sum_{n, m} I_{n, m} S_{n, m}(t) .
\end{aligned}
$$

1) Theorem 1: The Lyapunov function $E(t)$ of the VTF is bounded by $E_{\max }$ when a supplied voltage source is bounded.

Proof: To prove that $E(t)$ is bounded, we first examine $h\left(S_{n, m}\right)$. Since we assumed that the supplied voltage source is bounded, if we prove that the differential of $h\left(S_{n, m}\right)$ with respect to $S_{n, m}$ is bounded, we can conclude that $h\left(S_{n, m}\right)$ is bounded. From (10), we obtain a differential of $h\left(S_{n, m}\right)$ with respect to $S_{n, m}$ as

$$
\begin{aligned}
h^{\prime}\left(S_{n, m}(t)\right)= & \sum_{C(k, l) \in N_{r}(n, m)} G\left(N_{r}(n, m), \overrightarrow{v(t, x, y)}\right) \\
& \times(q / k T) e^{q\left(N_{r}(n, m)-I_{r} R-S_{n, m}\right) / k T} I_{s} .
\end{aligned}
$$

In (32), $h^{\prime}\left(S_{n, m}(t)\right)$ is bounded with given bounded $S_{n, m}$. Therefore, it is obvious that $h\left(S_{n, m}\right) h\left(S_{k, l}\right)$ is also bounded. To prove that $f\left(S_{n, m}\right)$ is bounded, we obtain $f^{\prime}\left(S_{n, m}\right)$ from (9) as

$$
\begin{aligned}
f^{\prime}\left(S_{n, m}\right)= & b \exp \left(b n_{2} S_{n, m}\right) F n_{2} \\
& +\frac{\left[\alpha \beta\left(\pi / 2+\arctan \left(C-n_{2} S_{n, m} / D\right)\right)\right]}{1+b \exp \left(B-C+n_{2} S_{n, m}\right)}-\gamma
\end{aligned}
$$

where

$$
\begin{aligned}
\alpha= & A\left[1+b \exp \left(B-C-n_{2} S_{n, m}\right)\right] \\
\beta= & \frac{b \exp \left(B-C+n_{2} S_{n, m}\right)}{1+b \exp \left(B-C-n_{2} S_{n, m}\right)} \\
& +b \exp \left(B-C-n_{2} S_{n, m}\right) \\
& \times \frac{\left[1+b \exp \left(B-C+n_{2} S_{n, m}\right)\right] n_{2}}{\left[1+b \exp \left(B-C-n_{2} S_{n, m}\right)\right]^{2}}
\end{aligned}
$$

and $r$ is given in (36), shown at the bottom of the page, where $b$ is $q / k T$. In (33), all the exponential functions are bounded with the given function $S_{n, m}$, which is bounded. Therefore, it is proven that $f\left(S_{n, m}\right)$ is bounded. In addition, since we have the bounded function $S_{n, m}$, the current source is bounded. Hence, $I_{n, m}$ is bounded. From the fact that every element of $E(t)$ is bounded, we can conclude that $E(t)$ is bounded.

2) Theorem 2: The differential of the Lyapunov function $E(t)$ of VTF is less than or equal to zero in the region where $f^{\prime}\left(S_{n, m}\right) \geq 0$, that is

$$
\frac{d E(t)}{d t} \leq 0, \quad f^{\prime}\left(S_{n, m}\right) \geq 0 .
$$

Proof: From (31), the differential of $E(t)$ with respect to time $t$ can be described by

$$
\begin{aligned}
\frac{d E(t)}{d t}= & -\sum_{n, m} \frac{d f\left(S_{n, m}(t)\right)}{d S_{n, m}(t)} \frac{d S_{n, m}(t)}{d t} h\left(S_{k, l}(t)\right) \\
& +\sum_{n, m} \frac{d f\left(S_{n, m}(t)\right)}{d S_{n, m}(t)} \frac{d S_{n, m}(t)}{d t} f\left(S_{n, m}(t)\right) \\
& +\sum_{n, m} I_{n, m} \frac{d f\left(S_{n, m}(t)\right)}{d S_{n, m}(t)} \frac{d S_{n, m}(t)}{d t} f\left(S_{n, m}(t)\right) \\
= & -\sum_{n, m} \frac{d f\left(S_{n, m}(t)\right)}{d S_{n, m}(t)} \frac{d S_{n, m}(t)}{d t} \\
& \times\left(h\left(S_{k, l}(t)\right)-f\left(S_{n, m}(t)-I_{n, m}\right)\right. \\
= & -\sum_{n, m} \frac{d f\left(S_{n, m}(t)\right)}{d S_{n, m}(t)}\left(\frac{d S_{n, m}(t)}{d t}\right)^{2} C .
\end{aligned}
$$

Since we can assume that $C$ is positive in physical meaning, the polarity of $E(t) / d t$ depends on $f^{\prime}\left(S_{n, m}\right)$. Assuming that $f^{\prime}\left(S_{n, m}\right) \geq 0$ leads to $E(t) / d t \leq 0$. From this theorem, the VTF is stable in a limited region where $f^{\prime}\left(S_{n, m}\right) \geq 0$.

However, our proposed circuit is stable ultimately. If the initial condition of $f^{\prime}\left(S_{n, m}\right)<0, S_{n, m}$ enters the positive differential resistive region, where $f^{\prime}\left(S_{n, m}\right) \geq 0$ due to the external current source. The external current source gives a driving force to $S_{n, m}$ from the negative differential region to the positive differential region.

3) Corollary 1: The output system is stabilized when the VTF is stabilized.

Proof: Assume that the Lyapunov function of the output system is $V(t)$. From (15), the stability of $g\left(O_{n, m}(t)\right)$ is affected only by $O_{n, m}$, since we know that $S_{n, m}$ is a stable function. As a result, the stability of the output system can be examined by the same procedure as that of the VTF. This leads to the same condition of stability, that is, the output system is stable only when $f\left(S_{n, m}\right) \geq 0$.

$$
\begin{aligned}
\gamma= & \frac{A n_{2} \ln \left[1+b \exp \left(B-C+n_{2} S_{n, m}\right) / 1+b \exp \left(B-C-n_{2} S_{n, m}\right)\right]}{D\left[1+\left(c-n_{2} S_{n, m}\right)^{2} / D^{2}\right]} \\
& -H n_{2} b \exp \left(n_{2} b S_{n, m}\right)
\end{aligned}
$$


4) Corollary 2: The VTF is asymptotically stable.

Proof: From Theorems 1 and 2, and Corollary 1, there exists any initial value of $S_{n, m}$ that satisfies the condition below

$$
\left\|S_{0,0}\right\|<\delta \Longrightarrow\left\|S_{n, m}\left(t, t_{0}, S_{0,0}\right)\right\|<\varepsilon \quad \forall t \geq t_{0} .
$$

In addition, it is attractive so that there exists a number $\delta>0$ such that, for all $t_{0} \geq 0$, it can be described by

$$
\left\|S_{0,0}\right\|<\delta \Longrightarrow\left\|S_{n, m}\left(t, t_{0}, S_{0,0}\right)\right\|<0, \quad t \rightarrow \infty .
$$

However, the VTF does not satisfy the previous condition if we assume an infinite voltage source. Because the infinite voltage source can generate infinite current source, the Lyapunov function $E(t)$ is unbounded from Theorem 1 .

\section{CONCLUSION}

In this paper, we have described a new VTF that is used for real-time motion estimation. Since the applications of the VTF necessitate tiny, energy-efficient, and speedy architecture, we have designed the new VTF based on the nanoelectronic architecture. In the nanoelectronic architecture, the VTF was implemented on a CNN based quantum dot structure. The experimental results show that the new VTF is 10, 100, and 1000 times better than conventional VTFs in area, power consumptions, and speed, respectively. Since the nanoelectronic architecture embraces nonlinear circuitry, a stability analysis was performed. Based on the analysis, we concluded that the new VTF is asymptotically stable, where the derivative of $I-V$ curve of the RTD with respect to $I$ is greater than or equal to 0 .

\section{REFERENCES}

[1] V. P. Roychowdhury, D. B. Janes, and S. Bandyopadhyay, "Collective computational activity in self-assembled arrays of quantum dots: A novel neuromorphic architecture for nanoelectronics," IEEE Trans. Electron Device, vol. 43, no. 10, pp. 1688-1699, Oct. 1996.

[2] V.P. Roychowdhury, D. B. Janes, and S. Bandyopadhyay, "Nanoelectronic architecture for boolean logic," Proc. IEEE, vol. 85, no. 4, pp. 574-588, Apr. 1997.

[3] E. H. Adelson, "Layered representation for image coding," Vis. Modeling Group, MIT Media Lab., Cambridge, Tech. Rep. 181, 1991.

[4] N. Franceschini, "Early processig of color and motion in a mosaic visual system,” Neuro-Sci.Res., vol. 2, pp. 17-49, 1985.

[5] A. B. Torralba and J. Herault, "An efficient neuromorphic analog network for motion estimation," IEEE Trans. Circuits Syst., vol. 46, no. 2, pp. 269280, Feb. 1999.

[6] L. Esaki and R. Tsu, "Superlattice and negative differenctial conductivity in semiconductors," IBM J. Res. Develop., vol. 14, no. 1, pp. 61-65, 1970.

[7] L. L. Chang, L. Esaki, and R. Tsu, "Resonant tunneling in semiconductor double barriers," Appl. Phys. Lett., vol. 24, no. 12, pp. 593-595, 1974.

[8] P. Mazumder, S. Kulkarni, M. Bhattacharya, J. P. Sun, and G. I. Haddad, "Digital circuit applications of resonant tunneling device," Proc. IEEE, vol. 86, no. 4, pp. 664-686, Apr. 1998.

[9] J. N. Schulman, H. J. D. L. Santos, and D. H. Chow, "Physics based RTD current-voltage equation," IEEE Electron Device Lett., vol. 17, no. 5, pp. 220-222, May 1996.

[10] T. P. E. Broekaert, B. Brar, J. P. A. van der Wagt, A. C. Seabaugh, T. S. Moise, F. J. Morris, E. A. Beam III, and G. A. Frazier, "A monolithic 4-bit 2-gsps resonant tunneling analog-to-digital converter," IEEE J. Solid-State Circuits, vol. 33, no. 9, pp. 1342-1349, Sep. 1998.

[11] J. P. Sun, G. I. Haddad, P. Mazumder, and J. N. Schulman, "Resonant tunneling diodes: Models and properties," Proc. IEEE, vol. 86, no. 4, pp. 641-660, Apr. 1998.

[12] R. D. Kenney, M. J. Schulte, and M. A. Erle, "A high-frequency decimal multiplier," in Proc. IEEE Int. Conf. Comput. Des., 2004, pp. 26-29.
[13] X. K. Yang, C. Zhu, Z. G. Li, X. Lin, and N. Ling, "Unequal packet loss resilience for MPEG-4 video over the internet," in Proc. ISCAS, 2000, vol. 2, pp. 832-835.

[14] A. Seabaugh, Z. Hu, Q. Liu, D. Rink, and J. Wang, "Silicon-based tunneling diodes and integrated circuits," in Proc. Int. Workshop Quantum Funct. Devices, 2000, pp. 5-8.

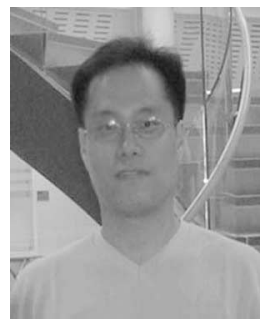

Woo Hyung Lee received the B.S. degree (summa cum laude) in electronic materials engineering from the Kwangwoon University, Seoul, Korea, in 1999, and the M.S. degree in materials science engineering from the Kwangju Institute of Science and Technology, Kwangju, Korea, in 2001. He is currently working toward the Ph.D degree in electrical engineering and computer science from the University of Michigan, Ann Arbor.

From 2001 to 2002, he was at Samsung Electronics, where he was engaged in hard disk drive development in the memory department. His current research interest include digital circuit research focusing on development of low-energy system and high-speed system.

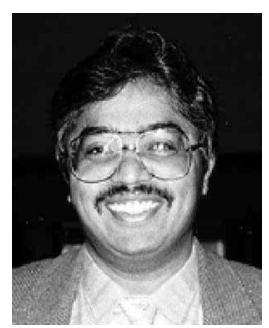

Pinaki Mazumder (S'84-M'88-SM'94-F'99) received the B.S.E.E. degree from the Indian Institute of Science, Bangalore, India, in 1976, the M.Sc. degree in computer science from the University of Alberta, Edmonton, AB, Canada, in 1985, and the Ph.D. degree in electrical and computer engineering from the University of Illinois, Urbana-Champaign, in 1987.

$\mathrm{He}$ is currently with the Department of Electrical Engineering and Computer Science, University of Michigan, Ann Arbor. He was a Research Assistant at the Coordinated Science Laboratory, University of Illinois, and Bharat Electronics, Ltd., where he was engaged in several types of analog and digital integrated circuits for consumer electronics products. During 1985 and 1986, he was a member of the Technical Staff of the Indian Hill Branch, Bell Laboratories, Napperville, IL. From 1996 to 1997, he was a visiting faculty member at Stanford University, Stanford, CA; University of California, Berkeley; and Nippon Telephone and Telegraph (NTT), Atsugi-shi, Japan. His current research interests include very-large-scale integration (VLSI) testing, physical design automation, and ultrafast circuit design. He is the author or coauthor of more than 160 papers published in various internatinal journals and proceedings of international conferences. He is the coauthor of Testing and Testable Design of High-Density Random-Access Memories (Kluwer, 1996), Semiconductor Memories: Testing and Reliability (EEE Comput. Soc., 1998), and Genetic Algorithms for VLSI Design, Layout, and Test Automation (Prentice-Hall, 1998). He holds two international patents. He has been involved in efforts in VLSI testing and built-in self-repair techniques, developed silicon compilers for RAM, ROM, and programmable logic array (PLA) with built-in self-repairable capabilities, worked in the area of VLSI physical design, and developed a suite of distributed place-and-route tools for VLSI and field-programmable gatearray (FPGA) chips. For six years, he has been an Integrated Circuit Designer for semiconductor companies. He currently leads ultrafast circuit design activities for nano and quantum electronic devices. He has successfully developed computer-aided design (CAD) tools for high-performance VLSI circuit simulation [NDR Simulation Program with Integrated Circuit Emphasis (SPICE)] and numerous circuit topologies for quantum MOS and other quantum-well devices. Several U.S. and Japanese semiconductor companies, including Texas Instruments Incorporated, Hughes Research Laboratory, Lockheed-Martin, NTT, and Nippon Electric Company (NEC), have collaborated with him on this research.

Dr. Mazumder was the Guest Editor of two special issues on emerging nanoelectronic technologies and their applications of the IEEE TRANSACTIONS ON VERY LARGE SCALE INTEGRATION (VLSI) SYSTEMS (December 1997) and the PROCEEDINGS OF THE IEEE (April 1998). He was the Guest Editor of the March 1993 Special Issue on Multimegabit Memory Testing of the IEEE DESIGN AND TEST OF COMPUTERs. He is on the Editorial Board of the PROCEEDINGS OF THE IEEE and is an Associate Editor of the IEEE TRANSACTIONS ON VERY LARGE SCALE INTEGRATION (VLSI) SYSTEMS. He was the Guest Editor of the June 1994 Special Issue on Memory Testing and Reliability of the Journal of Electronic Testing Theory and Applications. He was the recipient of the Digital's Incentives for Excellence Award, the BF Goodrich National Collegiate Invention Award, the National Science Foundation Research Initiation Award, and the Bell Northern Research Laboratory Faculty Award. 